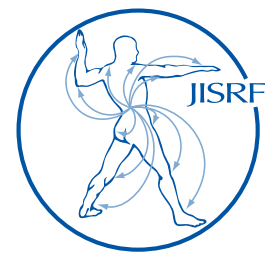

\title{
Restoration of Femoral Condylar Anatomy for Achieving Optimum Functional Expectations: Component Design and Early Results
}

\author{
Durbhakula $S^{1}, \operatorname{Rego} L^{1}$
}

\section{Abstract}

Background: Many total knee arthroplasty (TKA) systems are used across a variety of markets in which outcome will be influenced by patient morphology and normal activities of daily living, for that patient population. Femoral component sizing in primary total knee arthroplasty is of paramount importance for optimizing complication free post-operative function across all patients. The purpose of this study was to report the early results of a primary TKA system in support of the component design characteristics for achievement of increased functional expectations.

Methods: A prospective, continuous series of 176 primary posterior stabilized (PS) TKAs were performed in 172 patients by a single surgeon. Femoral component size distribution was assessed and all patients were followed for a minimum of two-years post-operatively. Total Hospital for Special Surgery (HSS) scores and range of motion (ROM) was assessed for the entire cohort and by gender.

Results: There were no patients lost to follow-up. Two patients required incision and drainage for superficial wound infection of the indicated knees. There was no radiographic evidence of component failure. As expected, femoral component size frequency use was skewed by gender with the larger sizes in males. There were no pre- or post-operative clinical or functional differences by gender and at the recent follow-up (avg. 3.8 years). In addition, there was an average significant increase in change of HSS score $(\mathrm{p}<0.01)$ and $\mathrm{ROM}(\mathrm{P}<0.01)$ when compared to pre-operative baseline.

Conclusions: The design characteristic for component sizing and functional expectations were confirmed in the reported Western population cohort series. Further continued use and study of this primary TKA system is warranted across all ethnic cultures.

Keywords: Total Knee; Condylar Anatomy

Level of Evidence: AAOS Therapeutic Level III

1 Sridhar Durbhakula, MD; Laura F. Rego, BS Washington Joint Institute at OrthoBethesda, The Camalier Building, 10215 Fernwood Road, Suite 502, Bethesda, MD 20817

(Direct reprint requests to Sridhar Durbhakula)
(C) 2016 Durbhakula, Rego. All rights reserved.

Reconstructive Review is a peer-reviewed, open-access orthopaedic journal devoted to publishing OPEN ACCESS papers in the area of reconstructive arthroplasty. Authors retain copyright and grant the journal

right of first publication with the work. Reconstructive Review follows the Creative Commons Attribution-NonCommercial CC BY-NC. This license allows anyone to download works, build upon the material, and share them with others for non-commercial purposes as long as they credit the senior author, Reconstructive Review, and the Joint Implant Surgery \& Research Foundation (JISRF). 


\section{Introduction}

Achieving successful expected outcomes following primary total knee arthroplasty (TKA) requires a balance of simultaneously occurring variables including surgical technique, component size availability and component design characteristics, each influenced by patient gender and anthropomorphic differences. In addition to relieving pain and creating a durable prosthetic composite, restoration of full function is a goal being pushed to its limits. Various TKA component designs have been introduced to address the need for increased flexion up to and beyond 140 degrees. However, most high-flexion component systems are based on standard implant design parameters that were engineered from Western patient morphology data leading to compromises regarding sizing and functional issues in the Middle-Eastern and Pan-Asian patient populations, and across socio-religious functional expectations. [1,5,9,10]

Intra-operative techniques for TKA, to accommodate component sizing availability across standard TKA components, are common for achieving acceptable joint reconstruction outcomes. For example, medial-lateral size availability, especially in smaller component offerings for women creates a marked overhang situation that may result in subsequent complications involving soft-tissue impingement, increased post-operative pain and decreased function. [6,7] In all cases, achieving increased ROM is balanced with component sizing and increased bone resection to accommodate component thickness.

Patient expectations regarding socio-religious needs such as prolonged deep-flexion, sitting cross-legged and squatting requires greater understanding of TKA versus normal knee kinematics. Increasing demands for greater knee function is a necessary outcome of western patients. [9] Engineering of TKA component dynamics is of paramount importance to successfully address the wide variety of patient anatomical needs and functional expectations. However, conflicting reports exist regarding high flexion TKA design and achieving flexion normally seen in standard posterior stabilized TKA systems [2] while other authors report maintenance or decrease in ROM following high flexion TKA. [8]

The purpose of this single surgeon, non-randomized, prospective case series of TKA patients was to report the early results of the Freedom ${ }^{\circledR}$ Total Knee system in support of the component design characteristics and achievement of early flexion expectations.

\section{Materials and Methods}

Between November 2010 and December 2013, the senior author performed 176 consecutive primary TKAs in 172 patients utilizing the posterior stabilized (PS) Freedom Total Knee ${ }^{\circledR}$ system (MAXX Orthopedics, Inc., Plymouth Meeting, Pennsylvania) (Figure 1). The Freedom Total Knee system is manufactured from cast cobalt chromium
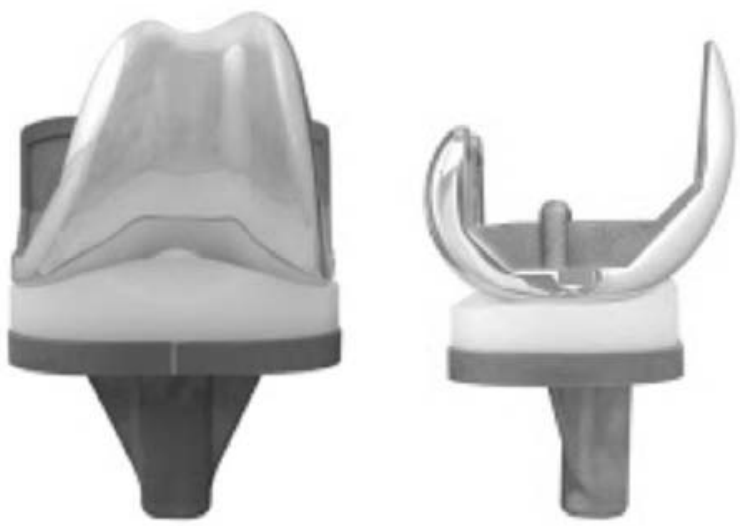

Figure 1. Anterior-Postetior and Lateral views of the Posterior Stabilized (PS) Freedom Total Knee® system (MAXX Orthopedics, Inc., Plymouth Meeting, Pennsylvania).

(ASTM F-75 CoCrMo), and the articular bearing surfaces use ram-extruded UHMWPE (GUR 1020). The Freedom Total Knee system was also designed with the intent to address bone conservation while permitting optimal high-flexion motion up to 155 degrees, dependent on the patient's anatomy and cultural activities of daily living, such as frequent and prolonged squatting and kneeling. To achieve high-flexion, the femoral component was engineered utilizing a multi-radius design in which seven tangential radii were incorporated to accommodate changes in rollback across the available surface through the transition from walking through deep flexion (Figure 2). In addition, development of femoral component sizing was optimized to include the anthropomorphic dimensions of Western and Pan-Asian patient populations.

There were 129 females (75.0\%) and 43 males (25.0\%) with an average patient age at surgery of $69.7 \pm 7.6$ years (range: 52.3 years to 98.6 years). The average age of the female patient (69.3 years) when compared to the male patients ( 70.7 years) was not significantly different $(p=0.311)$ (Table 1) In this continuous series, four female patients underwent bilateral TKA under the same anesthesia. The preoperative diagnosis was predominately degenerative joint disease (DJD) in 170 knees (96.6\%) and rheumatoid arthritis (RA) in 6 knees $(3.4 \%)$. The surgical side was evenly distributed across all patients with 86 left (48.9\%) and 90 right (51.1\%) knees (Table 1). 


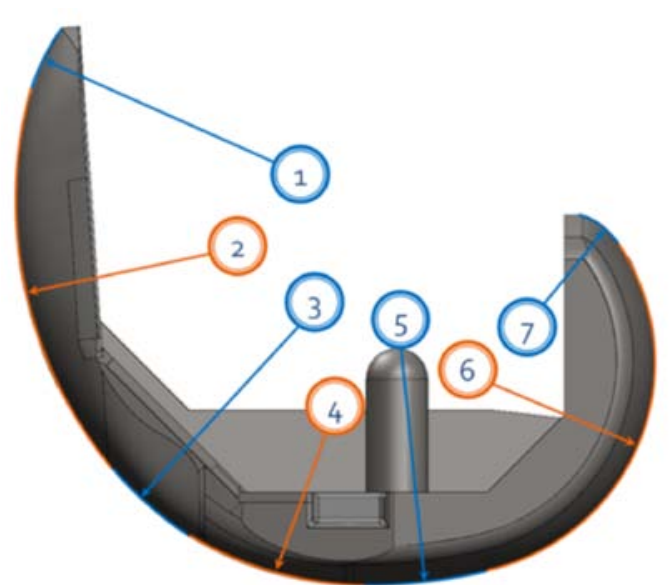

Figure 2. Multi-radius design of the Freedom Knee in which seven tangential radii were incorporated to accommodate changes in rollback across the available surface through the transition from walking through deep flexion. Radii 1,2 and 3 manage patellofemoral contact, and radii 4, 5, 6 and 7 control femoral rollback and flexion.

Table 1. Patient Demographics - there was no statistical difference in patient age at $p=0.311$. Also, there were 172 patients with $176 \mathrm{TKAs}$ (4 bilateral cases).

\begin{tabular}{|l|c|c|c|}
\hline & n & Males & Females \\
\hline $\begin{array}{l}\text { Total } \\
\text { Patients }\end{array}$ & $172(100 \%)$ & $43(25.0 \%)$ & $129(75.0 \%)$ \\
\hline $\begin{array}{l}\text { Average } \\
\text { Age }\end{array}$ & $69.7 \pm 7.9$ years & $70.7 \pm 7.3$ years & $69.3 \pm 7.7$ years \\
\hline Side & $\mathrm{R}=86(48.9 \%)$ & & \\
\hline $\begin{array}{l}\text { Pre- } \\
\text { Operative } \\
\text { Diagnoses }\end{array}$ & $\mathrm{L}=90(51.1 \%)$ & & \\
\hline DJD & $170(96.6 \%)$ & & \\
\hline RA & $6(3.4 \%)$ & & \\
\hline
\end{tabular}

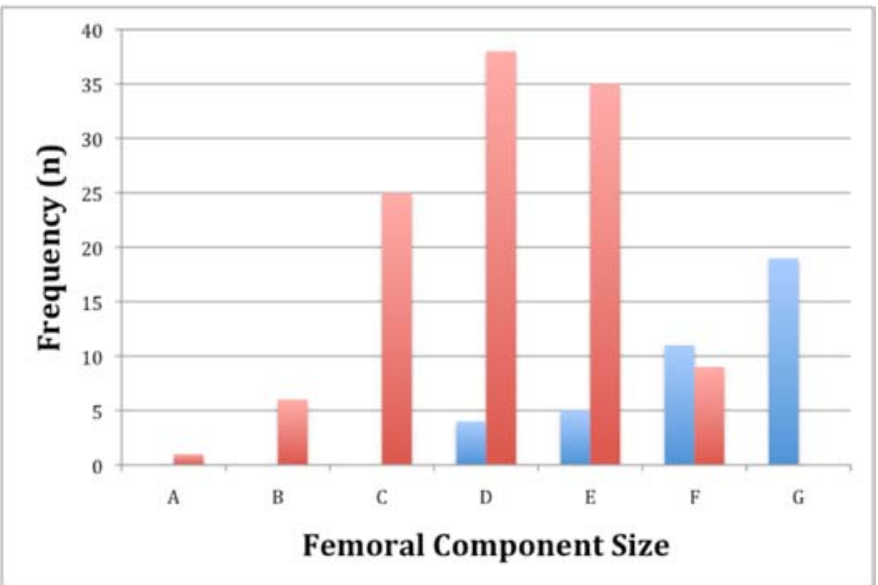

Figure 3. Distribution of component size by gender.

All patient data and radiographic information was deidentified to maintain patient confidentiality and compiled using Microsoft Excel software (Microsoft, Corp., Redmond, WA). Standard descriptive statistics were used to summarize the population and group comparisons were performed using Student's t-test with a level of significance at $\mathrm{p}<0.05$.

\section{Results}

All patients had a minimum follow-up of 2-years with an average time to follow-up of $3.8 \pm 0.9$ years (range: 2.2 years to 5.3 years). During the follow-up period there were no patients lost to follow-up and no failures. Two patients $(1.1 \%)$ required subsequent incision and drainage surgery for superficial wound infections. Following these procedures both patients went on to successful clinical and functional outcomes. The frequency of femoral component size use was different between genders and was skewed smaller for female patients versus male patients (Figure 3). There was no radiographic evidence of component loosening, osteolysis or failure in any patient (Figures 4A-D).

The pre-operative Hospital for Special Surgery (HSS) score was $49.2 \pm 5.7$ (range: 40.0 to 65.0 ), which significantly improved to an average of $88.4 \pm 3.6$ (range: 80.0 to 95.0) $(\mathrm{p}<0.001)$. There was no statistical difference in preoperative $(\mathrm{p}=0.208)$, post-operative $(\mathrm{p}=0.939)$ or change in HSS ( $p=0.296$ ) by gender. Functionally, the pre-operative range of motion $(\mathrm{ROM})$ was $113.8 \pm 6.1$ degrees (range: 95 degrees to 125 degrees), which improved to an average post-operative ROM of $128.5 \pm 4.1$ degrees (range: 110 degrees to 140 degrees) at the most recent follow-up. The change in ROM was statistically significant at $\mathrm{p}<0.01$. There was no statistical difference in pre-operative $(\mathrm{p}=0.566)$, post-operative $(\mathrm{p}=0.702)$ or change $(\mathrm{p}=0.484)$ in ROM by gender. All pre- and post-operative HSS and ROM data is summarized in Table 2.

\section{Discussion}

We present the minimum 2-year early results of a single surgeon, non-randomized, consecutive, prospective case series of patients receiving the Freedom Total Knee system for primary TKA. The results reported show significant improvement in HSS scors and achievement of increasedflexion thus confirming the component design characteristics incorporated by the manufacturer.

Dependent on anthropomorphic variables such as gender and ancestry, many femoral component offerings for primary TKA require a combination of compromising variables including component size and the amount of bone re- 

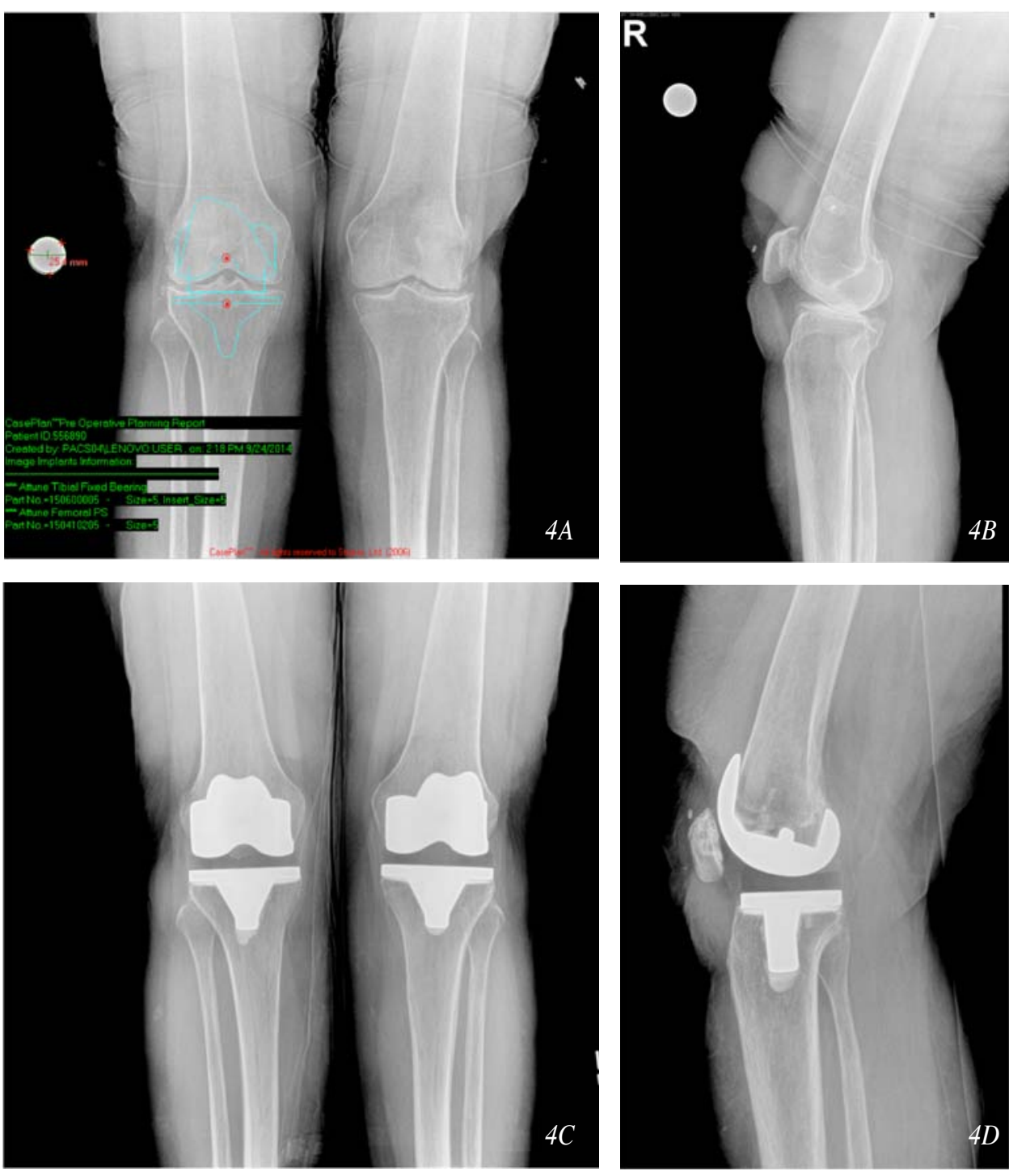

Figures 4A-D. Pre- and post-operative Anterior-Postetior and Lateral radiographs of the Posterior Stabilized (PS) Freedom Total Knee® system (MAXX Orthopedics, Inc., Plymouth Meeting, Pennsylvania).

moval necessary. Optimizing femoral component size for each patient contributes to maximizing outcome and avoiding related complications. However, various authors have reported differences in distal femoral anthropometry across cultures $[4,11]$ with possible soft-tissue and functional complications related to undersizing or overhanging the femoral component. Ho, et al, studied three different commercially available TKA components and reported consistent medial-lateral overhang in resected knees in the Chinese patient population. [4] Similarly in Indian women, Vaidya, et al, reported similar sizing issues in the medial-lateral aspect as well as in the anterior-posterior plane. Interestingly, standard femoral components were more compatible in Indian men. [11] Hitt, et al, reported the comparison of a cohort of patients in which distal fem-

Table 2. Patient Outcomes - Between genders there was no statistical difference in pre-operative ( $p=0.208)$ or post-operative $(p=0.939)$ HSS or pre-operative ( $p=0.566)$ or post-operative $(p=0.702)$ ROM. Change in HSS and ROM from pre-to post-operative follow-up was statistically siginificant (HSS: $p<0.001$, ROM $p<0.01$ ).

\begin{tabular}{|l|c|c|c|}
\hline & $\begin{array}{c}\text { Total } \\
\text { Population }\end{array}$ & Males & Females \\
\hline $\begin{array}{l}\text { Avg Follow- } \\
\text { Up }\end{array}$ & $3.8 \pm 0.9$ years & & \\
\hline $\begin{array}{l}\text { Pre-Op } \\
\text { HSS }\end{array}$ & $49.2 \pm 5.7$ & $48.3 \pm 5.6$ & $49.5 \pm 5.7$ \\
\hline $\begin{array}{l}\text { Post-Op } \\
\text { HSS }\end{array}$ & $88.4 \pm 3.6$ & $88.4 \pm 4.2$ & $88.4 \pm 3.4$ \\
\hline $\begin{array}{l}\text { Pre-Op } \\
\text { ROM }\end{array}$ & $113.8 \pm 6.1$ & $114.3 \pm 6.0$ & $113.7 \pm 6.2$ \\
\hline $\begin{array}{l}\text { Post-Op } \\
\text { ROM }\end{array}$ & $128.5 \pm 4.1$ & $128.3 \pm 3.8$ & $128.5 \pm 4.3$ \\
\hline
\end{tabular}
oral morphology was directly compared to five different commercially available primary TKA systems.(Hitt et al., 2003) The data revealed significant overhang of the femoral components in women $(\mathrm{p}<0.0001)$ while in men there was complimentary sizing match $(\mathrm{p}<0.79)$ when compared to patient morphology. The authors concluded that there are significant variations in the femoral aspect ratio dimensions of the components when compared to anatomic knee morphology. These variations may compromise the balance between component size and optimum post-operative function. In all three reported studies the Pan-Asian patient has significant morphological differences than the Western population that common TKA devices are designed against. The Freedom Total Knee system was designed for bridging the size requirements across Western and Asian population anthropomorphic variations without compromising functional needs. Mid-range component design in the medial-lateral and anterior-posterior distal femoral aspects allows for a transitional size and shape for use across gender and various global populations. While the results 
of the presented study reveal gender-specific differences in component sizing, similar clinical and functional outcomes at an average of 2-years follow-up were observed independent of gender.

Restoration of activities of daily living (ADL) following primary TKA is a primary contributing factor in overall patient satisfaction and depends on pre-operative patient expectations. In a study of Western patients, Weiss, et al, found that post-TKA patients were unable to perform deep flexion activities and were discontented with the limits involving deep flexion activities. [12] Besides the relief of pain, achieving early and full ROM is a significant hurdle for maximizing patient satisfaction. Conversely, using a component designed for deep flexion, Tarabichi, et al, reported results of achieving high flexion following TKA in Muslim patients requiring achievement of a fully flexed knee for lifestyle needs including praying, social encounters, dining and bathroom use. [9] This did not lead to an increase in post-operative complications and validates the ability to achieve increased kinematics without sacrificing the prosthetic composite. In an effort to conserve bone while achieving a condylar profile that allows for full ROM, the Freedom Total Knee system was designed to accommodate changes in the femoral condylar radii of curvature based on the degree of flexion change and the amount of rollback needed through ROM while minimizing bone resection. The femoral condylar profile of the Freedom Total Knee System integrates seven radii to manage articulation across full ROM, allowing for a large radii or arc to inhibit significant rollback in normal gait and smaller radii for greater rollback as flexion increases into deep, full flexion with smooth transition across the entire femoral condylar profile. The results of the reported study show that early ROM across our patient cohort can be achieved while avoiding common complications associated with component sizing and early restoration of function.

When interpreting the reported results one must consider that this is a single surgeon, non-randomized case series. However, the study included a large cohort $(n=172)$ of continuous non-selected series of patients without loss to follow-up for any reason. There was an imbalance of cases by gender (females $n=129$, males $n=43$ ), and while gender had an affect on femoral component sizing, this variable did not influence outcome regarding ROM or total HSS. Also, the patients included were of a Western ancestry population, so no comparative assessments to MiddleEastern or Pan-Asian populations were available.

In conclusion, the reported study of patients receiving the Freedom Total Knee system for primary TKA support the design considerations for anthropomorphic considerations while achieving an increased degree of ROM while minimizing bone resection. Further continued use and study is warranted to confirm achieving similar results across surgeons and multiple ancestral populations.

\section{Disclosure}

One or more of our authors have disclosed information that may present potential for conflict of interest with this work. For full disclosures refer to last page of this journal.

\section{Acknowledgment}

The authors wish to thank Robert Eberle for his assistance with the preparation of this manuscript.

\section{References}

1. Acker, S. M., Cockburn, R. A., Krevolin, J., Li, R. M., Tarabichi, S., \& Wyss, U. P. (2011). Knee kinematics of high-flexion activities of daily living performed by male Muslims in the Middle East. J Arthroplasty, 26(2), 319-327. doi:10.1016/j. arth.2010.08.003

2. Hamilton, W. G., Sritulanondha, S., \& Engh, C. A., Jr. (2011). Results of prospective, randomized clinical trials comparing standard and high-flexion posterior-stabilized TKA: a focused review. Orthopedics, 34(9), e500-503. doi:10.3928/01477447-20110714-47

3. Hitt, K., Shurman, J. R., 2nd, Greene, K., McCarthy, J., Moskal, J., Hoeman, T., \& Mont, M. A. (2003). Anthropometric measurements of the human knee: correlation to the sizing of current knee arthroplasty systems. J Bone Joint Surg Am, 85-A Suppl 4, 115-122.

4. Ho, W. P., Cheng, C. K., \& Liau, J. J. (2006). Morphometrical measurements of resected surface of femurs in Chinese knees: correlation to the sizing of current femoral implants. Knee, 13(1), 12-14. doi:10.1016/j.knee.2005.05.002

5. Lee, B. S., Chung, J. W., Kim, J. M., Kim, K. A., \& Bin, S. I. (2013). High-flexion prosthesis improves function of TKA in Asian patients without decreasing early survivorship. Clin Orthop Relat Res, 471(5), 1504-1511. doi:10.1007/s11999012-2661-4

6. Matsumoto, T., Kubo, S., Muratsu, H., Tsumura, N., Ishida, K., Matsushita, T., . . . Kuroda, R. (2011). Differing prosthetic alignment and femoral component sizing between 2 computer-assisted CT-free navigation systems in TKA. Orthopedics, 34(12), e860-865. doi: $10.3928 / 01477447-20111021-35$

7. Rosenstein, A. D., Veazey, B., Shephard, D., \& Xu, K. T. (2008). Gender differences in the distal femur dimensions and variation patterns in relation to TKA component sizing. Orthopedics, 31(7), 652.

8. Tanavalee, A., Ngarmukos, S., Tantavisut, S., \& Limtrakul, A. (2011). High-flexion TKA in patients with a minimum of 120 degrees of pre-operative knee flexion: outcomes at six years of follow-up. Int Orthop, 35(9), 1321-1326. doi:10.1007/ s00264-010-1140-3

9. Tarabichi, S., Tarabichi, Y., \& Hawari, M. (2010). Achieving deep flexion after primary total knee arthroplasty. J Arthroplasty, 25(2), 219-224. doi:10.1016/j. arth.2008.11.013

10. Uehara, K., Kadoya, Y., Kobayashi, A., Ohashi, H., \& Yamano, Y. (2002). Anthropometry of the proximal tibia to design a total knee prosthesis for the Japanese population. J Arthroplasty, 17(8), 1028-1032. doi:-

11. Vaidya, S. V., Ranawat, C. S., Aroojis, A., \& Laud, N. S. (2000). Anthropometric measurements to design total knee prostheses for the Indian population. J Arthroplasty, 15(1), 79-85

12. Weiss, J. M., Noble, P. C., Conditt, M. A., Kohl, H. W., Roberts, S., Cook, K. F., . Mathis, K. B. (2002). What functional activities are important to patients with knee replacements? Clin Orthop Relat Res(404), 172-188. 